\title{
MONTE CARLO SIMULATION OF A MAMMOGRAPHIC TEST PHANTOM
}

\author{
R. A. Hunt ${ }^{1}$, D. R. Dance ${ }^{1, *}$, M. Pachoud ${ }^{2}$, G. Alm Carlsson ${ }^{3}$, M. Sandborg ${ }^{3}$, G. Ullman ${ }^{3}$ \\ and F. R. Verdun ${ }^{2}$ \\ ${ }^{1}$ Department of Physics, The Royal Marsden Hospital NHS Foundation Trust, London SW3 6JJ, UK \\ ${ }^{2}$ University Institute for Applied Radiophysics, Grand-Pré 1 CH-1007 Lausanne, Switzerland \\ ${ }^{3}$ Department of Radiation Physics, IMV, Faculty of Health Sciences, Linköping University, \\ SE-58185 Linköping, Sweden
}

\begin{abstract}
A test phantom, including a wide range of mammographic tissue equivalent materials and test details, was imaged on a digital mammographic system. In order to quantify the effect of scatter on the contrast obtained for the test details, calculations of the scatter-to-primary ratio (S/P) have been made using a Monte Carlo simulation of the digital mammographic imaging chain, grid and test phantom. The results show that the S/P values corresponding to the imaging conditions used were in the range 0.084-0.126. Calculated and measured pixel values in different regions of the image were compared as a validation of the model and showed excellent agreement. The results indicate the potential of Monte Carlo methods in the image qualitypatient dose process optimisation, especially in the assessment of imaging conditions not available on standard mammographic units.
\end{abstract}

\section{INTRODUCTION}

The need for image quality assessments and optimisation in mammography is well established and is often based on the use of test phantoms ${ }^{(1,2)}$. One limitation of most of these test phantoms is that they are of uniform thickness and composition. Thus, they do not address the issue of the variation of image quality with tissue composition. In this work a test phantom has been designed to allow an objective assessment of image quality in conventional and in digital mammography, taking into account the dynamic range of the exposure ${ }^{(3)}$. The purpose of this test phantom is to assess the optimum between dose and image quality.

Another approach for improving the balance between the radiological risk and the image quality is the use of Monte Carlo simulations. They have been widely used in the field of diagnostic radiology to estimate doses delivered to patients. In the early days, to render calculations tractable, organs were represented by simple volumes such as spheres or cylinders, and a very simple model of the breast was used for mammography ${ }^{(4)}$. Major improvements of the computing power now allow the assessment of patient dose using voxel phantoms where the shapes and positions of the organs are realistic ${ }^{(5)}$. In the framework of patient dose optimisation, Monte Carlo simulations have been extensively used to estimate scatter-to-primary ratio (S/P), in particular to improve the antiscatter grid performances ${ }^{(6)}$. Finally, Monte Carlo simulations are now being used to simulate the whole radiological chain (i.e. from

*Corresponding author: david.dance@rmh.nthames.nhs.uk the X-ray production to the raw data images) ${ }^{(7,8)}$. These capabilities (precise dose assessment and raw image production) widen the domain of optimisation since no limitation concerning the availability of the technology exists.

In an initial study of images obtained with the test phantom, measurements of the contrast of test details were compared with analytical calculations based on the attenuation of primary photons, but the effects of scatter were not properly taken into account because of lack of appropriate data. In order to provide details of the effect of scattered radiation on the contrast, a voxelised model of the phantom has been constructed and Monte Carlo calculations made of the S/P obtained both with and without an antiscatter grid. In addition, a comparison of measured and calculated pixel values in digital images of the phantom has provided a validation of the computer program.

\section{MATERIALS AND METHODS}

The test phantom used in this study has been fully described elsewhere ${ }^{(3)}$ and was manufactured by CIRS (Computerized Imaging Reference Systems, Norfolk Virginia 23513, USA). It is made of breast tissue equivalent material and has a similar shape to a compressed breast (Figure 1). A 35-mm base layer of 50:50 glandular/adipose tissue equivalent is surrounded by a $5-\mathrm{mm}$ shell of $100 \%$ adipose equivalent material to simulate the absorption of the skin (total phantom thickness $45 \mathrm{~mm}$ ). Three areas within the test phantom simulate the absorption of $100 \%$ glandular, 50:50 glandular/adipose and 100\% adipose tissue. Low contrast targets next to the 

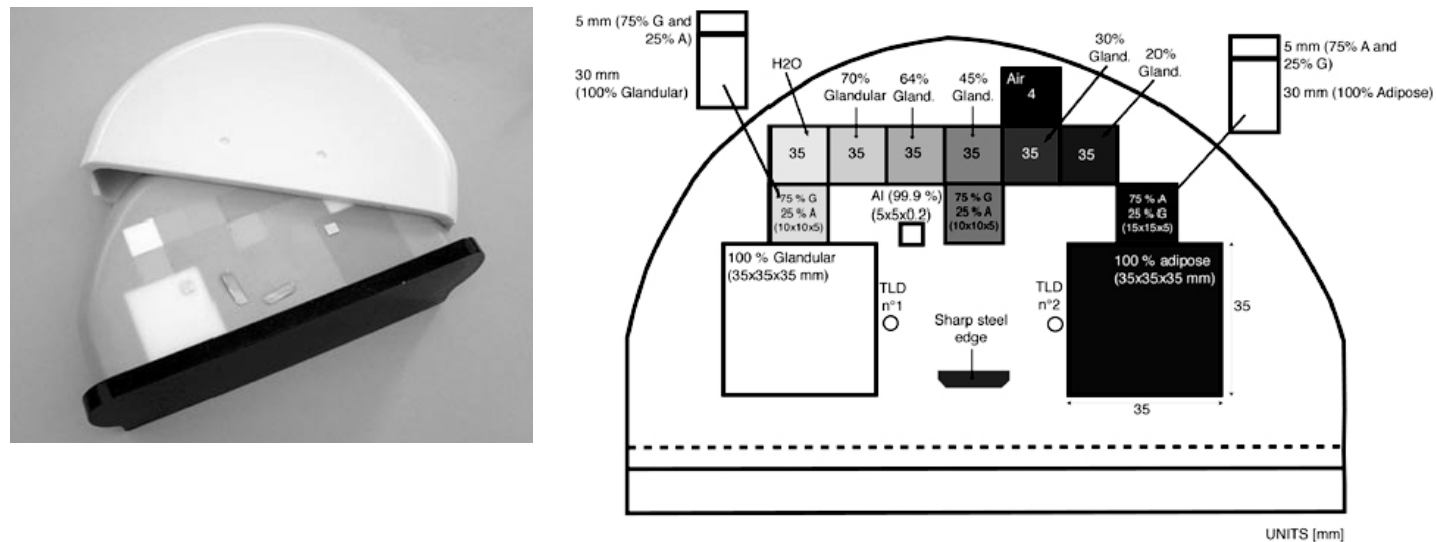

Figure 1. Picture of the test phantom showing the different tissue equivalent regions and the removable adipose shell and its original schematic representation. (As shown in Figure 2a, the manufactured test phantom has been slightly modified from the original schematic representation).
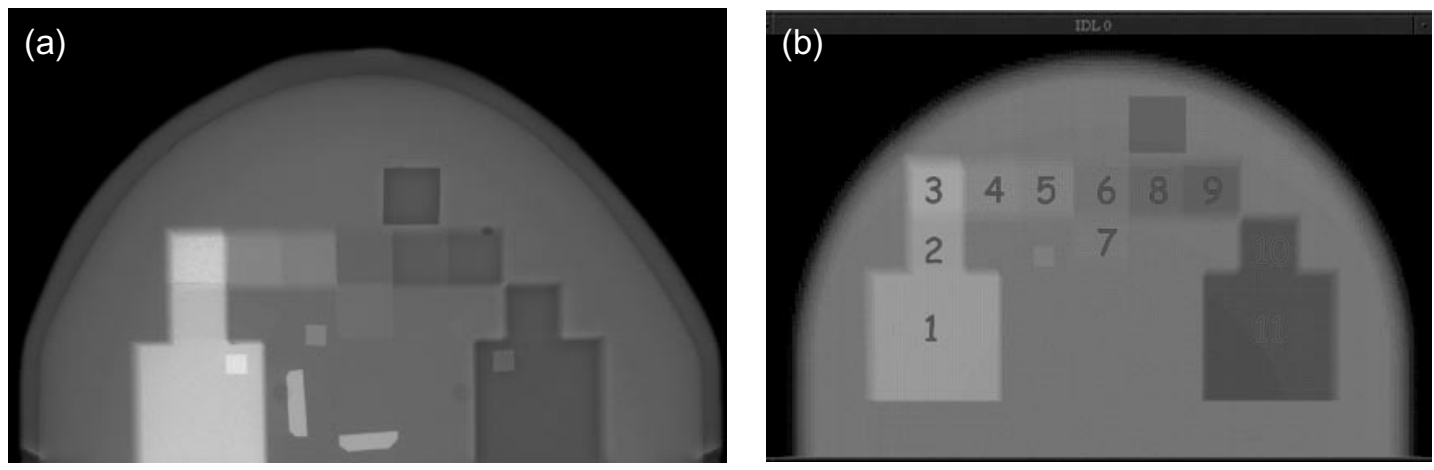

Figure 2. (a) Radiograph of the test phantom using a GE Senographe 2000D digital mammography unit at $28 \mathrm{kV}$ using a Mo-Mo anode-filter combination. (b) Image produced by Monte Carlo simulation with the schematic representation shown in Figure 1.

$100 \%$ glandular and 50:50 glandular/adipose areas are produced by 5-mm-thick slabs of 75:25 glandular/ adipose tissue equivalent material. A 5-mm slab of 25:75 glandular/adipose tissue gives a third low contrast target next to the $100 \%$ adipose area.

Digital images were acquired on a Senographe 2000D unit (General Electric, USA) using a high voltage of $28 \mathrm{kV}$, a tube current-exposure time product of $45 \mathrm{mAs}$ and a Mo-Mo anode-filter combination (exposure parameters representative of those used with a conventional mammography unit).

The Monte Carlo program used to simulate the phantom is based on a code developed previously for the simulation of mammography using simple phantoms ${ }^{(7)}$. The program has been modified to incorporate voxel phantoms and can calculate both pixel values and the noise per pixel ${ }^{(8)}$. For the latter purpose, only quantum mottle is included and system unsharpness is ignored. The simulation includes the CsI receptor used in the GE 2000D mammography unit and the mammographic antiscatter grid. The X-ray spectrum was matched to that used for the experimental measurements. The voxel phantom was constructed mathematically from the design specification of the phantom, although the sharp steel edge and TLDs were omitted. The program makes separate calculations of the energy deposited per pixel from primary and secondary photons and can thus give information on the variation of the $\mathrm{S} / \mathrm{P}$ ratio and of the pixel value across the image.

\section{RESULTS AND DISCUSSION}

Figure $2 \mathrm{a}$ and $\mathrm{b}$ present the image obtained with the mammography unit and the calculated image using the Monte Carlo program, respectively. These figures show that the test phantom developed allows image quality parameter assessment for a wide range 
R. A. HUNT ET AL.

Table 1. S/P values calculated with the Monte Carlo method.

\begin{tabular}{lcc}
\hline $\begin{array}{l}\text { Number of } \\
\text { the area }\end{array}$ & $\begin{array}{c}\text { S/P (no } \\
\text { antiscatter grid) }\end{array}$ & $\begin{array}{c}\text { S/P (with } \\
\text { antiscatter grid) }\end{array}$ \\
\hline 1 & 0.616 & 0.1008 \\
2 & 0.626 & 0.1027 \\
3 & 0.759 & 0.1258 \\
4 & 0.582 & 0.1053 \\
5 & 0.570 & 0.0982 \\
6 & 0.528 & 0.0925 \\
7 & 0.561 & 0.0948 \\
8 & 0.476 & 0.0903 \\
9 & 0.472 & 0.0864 \\
10 & 0.450 & 0.0883 \\
11 & 0.465 & 0.0843 \\
\hline
\end{tabular}

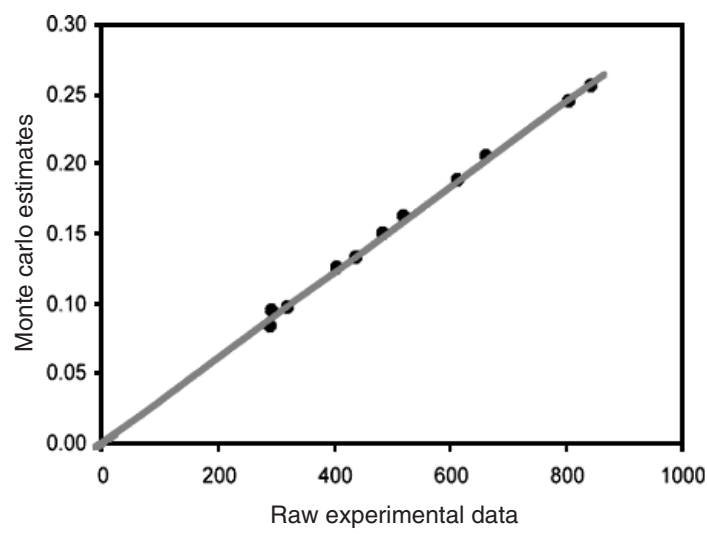

Figure 3. Relationship between measured pixel values on the radiograph of the test phantom and the energy imparted per pixel (arbitrary units) computed by the Monte Carlo model at various locations within the test phantom.

of tissue compositions in the same image. Moreover, the broad similarity of these two figures indicates the utility of the Monte Carlo approach. The differences between the two images are due to the fact that the initial design of the test phantom which has been used in the Monte Carlo simulations (Figure 1) has been slightly modified during the test phantom manufacturing. These differences have no major impact on the results, but will be accounted for in future calculations.

The S/P values, calculated by Monte Carlo in terms of energy imparted to the image detector and repeated with and without the antiscatter grid, are summarised in Table 1 . The areas where $\mathrm{S} / \mathrm{P}$ have been calculated have been numbered according to Figure 2b. As expected, the S/P without the grid in the $100 \%$ glandular part ( 0.616 in area 1$)$ is higher than in the $100 \%$ adipose part ( 0.456 in area 11$)$, in particular, because of its higher primary X-ray absorption properties compared with the fully adipose tissue equivalent area.

Figure 3 presents the relationship between the measured pixel values, at various positions, on the image obtained with the digital mammography unit and the pixel values calculated using the Monte Carlo methodology, both for a Mo-Mo spectrum at $28 \mathrm{kV}$. The calculations take account of both primary and scattered radiation. The data have been fitted to a straight line constrained to pass through the origin as both calculated and experimental pixel values are zero when there is no exposure. The excellent correlation obtained (Pearson coefficient $\mathrm{R}^{2}=0.9971$ ) is an encouraging demonstration of the proportionality of experimental and calculated pixel values and validation of the computer model.

\section{CONCLUSIONS}

The results of this work have allowed us to obtain values of the $\mathrm{S} / \mathrm{P}$ for the Lausanne mammographic phantom both with and without an antiscatter grid. In spite of relatively large differences in breastequivalent tissue compositions within the test phantom, the $S / P$ variations at the entrance of the detector when the grid is used remain small. The results presented in this study and a related paper in this journal ${ }^{(8)}$ clearly demonstrate that Monte Carlo methods can be used to accurately simulate radiological imaging situations.

\section{ACKNOWLEDGEMENTS}

The authors gratefully acknowledge the financial support of the Swiss Federal Office for Education and Science (grant number 99.0739) and the EU Fifth Framework Research Programme (grant number FIGMCT 2000 00036).

\section{REFERENCES}

1. van Voudenberg, S., Thijssen, M. and Young, K. European guidelines for quality assurance in mammography screening. Third edition [Luxembourg: CEC (European Commission)] (1999). Report EUR no. 14821 (1999).

2. Hendrick, R. E. and Berns, E. A. Optimizing mammographic technique. In: Proceedings of the RSNA categorical course in breast imaging. Chicago, IL, pp. 79-89 (1999).

3. Pachoud, M., Lepori, D., Valley, J. F. and Verdun, F. R. Development of a test object to assess image quality in conventional and digital mammography, taking into account dynamic range. Phys. Med. Biol. 49, 5267-5281 (2004).

4. Dance, D. R. and Day, G. J. The computation of scatter in mammography by Monte Carlo methods. Phys. Med. Biol. 29, 237-247 (1984). 


\section{SIMULATION OF A MAMMOGRAPHIC TEST PHANTOM}

5. Zankl, M., Fill, U., Petoussi-Henss, N. and Regulla, D. Organ dose conversion coefficients for external photon irradiation of male and female voxel models. Phys. Med. Biol. 47, 2367-2385 (2002).

6. Sandborg, M., Dance, D. R., Alm Carlsson, G. and Persliden, J. Monte Carlo study of grid performance in diagnostic radiology: task dependent optimisation for screen-film imaging. Brit. J. Radiol. 67, 76-85 (1994).

7. Dance, D. R., Thilander, A. K., Sandborg, M., Skinner, C. L., Castellano, I. A. and Carlsson, G. A.
Influence of anodelfilter material and tube potential on contrast, signal-to-noise ratio and average absorbed dose in mammography: a Monte Carlo study. Brit. J. Radiol. 73, 1056-1067 (2000).

8. Hunt, R. A., Dance, D. R., Bakic, P. R., Maidment, A. D. A., Sandborg, M., Ullman, G. and Alm Carlsson, G. Calculation of the properties of digital mammograms using a computer simulation. Rad. Prot. Dosim. 114(1-3), 395-398, (2005). 